\title{
The RNA-Binding Protein ELAVL1 Regulates Hepatitis B Virus Replication And Growth of Hepatocellular Carcinoma Cells
}

Hiroaki Kanzaki

Chiba University

Tetsuhiro Chiba ( $\nabla$ chibat@chiba-u.jp )

Chiba University

Tatsuya Kaneko

Chiba University

Junjie Ao

Chiba University

Na Qiang

Chiba University

Yaojia Ma

Chiba University

Takamasa Ishino

Chiba University

Keita Ogawa

Chiba University

Miyuki Nakagawa

Chiba University

Kisako Fujiwara

Chiba University

Hidemi Unozawa

Chiba University

Terunao Iwanaga

Chiba University

Motoyasu Kan

Chiba University

Takafumi Sakuma

Chiba University

Naoto Fujita

Chiba University

Keisuke Koroki 
Chiba University

\section{Yuko Kusakabe}

Chiba University

Kazufumi Kobayashi

Chiba University

Naoya Kanogawa

Chiba University

\section{Soichiro Kiyono}

Chiba University

Masato Nakamura

Chiba University

\section{Takayuki Kondo}

Chiba University

\section{Tomoko Saito}

Chiba University

\section{Ryo Nakagawa}

Chiba University

\section{Sadahisa Ogasawara}

Chiba University

Ryosuke Muroyama

Chiba University

\section{Shingo Nakamoto}

Chiba University

\section{Tatsuo Kanda}

Nihon University School of Medicine

\section{Hitoshi Maruyama}

Juntendo University School of Medicine

Jun Kato

Chiba University

\section{Yoh Zen}

King's College Hospital

\section{Ai Kotani}

Tokai University

\section{Kazuma Sekiba}

The University of Tokyo

\section{Motoyuki Otsuka}

The University of Tokyo

\section{Masayuki Ohtsuka}

Chiba University 


\section{Naoya Kato}

Chiba University

\section{Research Article}

Keywords: immunoprecipitation, proteomic, Embryonic Lethal, hepatocellular carcinoma

Posted Date: September 15th, 2021

DOI: https://doi.org/10.21203/rs.3.rs-860409/v1

License: (c) (1) This work is licensed under a Creative Commons Attribution 4.0 International License. Read Full License 


\section{Abstract}

Previous RNA immunoprecipitation followed by proteomic approaches successfully demonstrated that Embryonic Lethal, Abnormal Vision, Drosophila-Like 1 (ELAVL1) interacts with hepatitis B virus (HBV)derived RNAs. Although ELAVL family proteins stabilize AU-rich element (ARE)-containing mRNAs, their role in HBV transcription remains unclear. This study conducted loss-of-function assays of ELAVL1 for inducible HBV-replicating HepAD38 cells and HBx-overexpressed HepG2 cells. In addition, clinicopathological analyses in primary hepatocellular carcinoma (HCC) surgical samples have also been conducted. Lentivirus-mediated short hairpin RNA knockdown of ELAVL 1 resulted in a decrease in both viral RNA transcription and production of viral proteins, including HBs and HBx. Cell growth in tetracycline-treated replication-stopping HepAD38 cells was more significantly impaired in ELAVL 1 knockdown than those in the control group, indicating that ELAVL1 is involved in proliferation not only by the suppression of HBV replication but also by other factors. Immunohistochemical analyses of 77 paired HCC surgical specimens have demonstrated that diffuse ELAVL1 expression was detected more frequently in HCC tissues (61.0\%) than in nontumor tissues (27.3\%). In addition, the abundant expression of ELAVL1 tended to affect postoperative recurrence in HBV-related HCC patients. In conclusion, ELAVL1 contributes not only to HBV replication but also to HCC cell growth. It may be a potent therapeutic target for HBV-related HCC treatment.

\section{Introduction}

Hepatitis B virus (HBV) infection is one of the global health concerns. Despite the existence of a prophylactic vaccine, approximately 240 million individuals worldwide are currently infected and at a high risk of developing cirrhosis and hepatocellular carcinoma (HCC) ${ }^{1-3}$. HBV virions contain partially doublestranded, relaxed circular DNA (3.2-kb genome in length), from which covalently closed circular DNA (cccDNA) is formed. HBV cccDNA is persistently present in the nucleus of hepatocytes in an episomal state and serves as a template for viral transcription ${ }^{4,5}$. It is known that five types of RNA are transcribed using cccDNA as a template ${ }^{6}$. There are two kinds of $3.5 \mathrm{~kb}$ mRNA, namely precore mRNA and pregenomic RNA. Precore mRNA translates $\mathrm{HBeAg}$, and pregenomic RNA translates $\mathrm{HBc}$ and polymerase. HBs antigens (large, middle, and small S proteins), which are envelope proteins, are translated from 2.4/2.1 kb mRNA. 0.7kb mRNA encodes an HBx protein that regulates transcription of viral mRNA from cccDNA. Previously, it was believed that HBV-derived RNAs were only templates for viral protein translation and intermediates for the viral DNA synthesis by reverse transcription for replication, but HBVderived RNAs have recently been found to also have crucial pathological functions ${ }^{7-9}$.

The currently available HBV therapeutics, including nucleos(t)ide analogs (NAs), efficiently suppress viral reverse transcription and thereby reduce serum levels of HBV-DNA. However, episomal viral cccDNA, as well as transcripts and proteins from cccDNA, maintain to be expressed during these treatments ${ }^{10-11}$. In this context, both cccDNA and transcripts and proteins from cccDNA might be a potential new therapeutic target. Sekiba et al. performed a comprehensive RNA precipitation assay to determine host proteins that 
interact with HBV-derived RNAs including the $S, X$, and core regions ${ }^{12}$. Consequently, they successfully identified 15 candidate proteins. Among them, the RNA-protein binding has been successfully confirmed by western blotting for six proteins, including ELAVL1 (also known as HuR). ELAVL1 is a ubiquitously expressed RNA-binding protein in many types of cells and plays an important role in mRNA stability regulation. The molecule binds to a specific single-stranded motif in AU-rich elements (ARE) primarily located in the $3^{\prime}$ untranslated regions of early response genes ${ }^{13}$. However, the role of ELAVL1 in not only HBV replication but also in cell growth remains unknown.

In this study, the effects of ELAVL1 on HBV-derived RNAs and proteins in loss-of-function assays using HepAD38 cells, which can induce HBV replication under tetracycline depletion and $H B x$-overexpressed HepG2 cells, have been examined. To unveil the association between ELAVL1 expression and clinicopathological features, paired HCC surgical specimens were subjected to immunohistochemical analyses for ELAVL1.

\section{Results}

\section{ELAVL1 expression in HCC cells with or without HBV replication}

HBV replication in HepAD38 cells could be controlled using tetracycline, which inhibits HBV transcription from the genome. As expected, the expressions of both total HBV mRNA and HBV 3.5-kb mRNA were strongly repressed under tetracycline treatment (Fig. 1A). ELAVL 1 mRNA expression in HepAD38 cells has been analyzed with or without HBV replication, respectively. Surprisingly, HBV replication caused by tetracycline depletion resulted in an increased ELAVL 1 mRNA expression (Fig. 1B). This indicates that HBV replication is closely associated with ELAVL1 expression.

\section{Stable knockdown of ELAVL1 in HCC cells}

Loss-of-function assay of ELAVL 1 was first conducted using two different shRNA targeting ELAVL1 (shELAVL 1-1 and sh-ELAVL 1-2). A lentiviral vector-expressed shRNA targeting /uciferase ( $L u C$ ) was used as a control. HepAD38 cells that stably expressed shRNA targeting ELAVL 1 or $L u c$ were successfully achieved by lentiviral transduction. Quantitative real-time PCR demonstrated that shRNAs against ELAVL1 significantly repressed ELAVL $1 \mathrm{mRNA}$ levels (Fig. 1C). Consistent with these findings, western blotting also demonstrated decreased ELAVL1 protein levels in the ELAVL 1 knockdowncells (Fig. 1D).

\section{The effect of ELAVL1 on HBV-derived RNAs and proteins}

To investigate the effect of ELAVL1 on HBV-derived RNAs, mRNAs extracted from ELAVL1knockdownHepAD38 cells were subjected to quantitative real-time PCR using specific primers for total HBV mRNA and HBV 3.5-kb mRNA (encoding HBV core and pregenomic RNA (pgRNA)).

ELAVL 1 knockdown resulted in a remarkable decrease in not only total HBV mRNA levels but also in HBV 3.5-kb mRNA levels (Fig. 2A). Next, the effect of ELAVL1on the production of HBs and HBx protein has been examined. Chemiluminescent enzyme immunoassay (CLEIA) demonstrated that secreted HBsAg 
levels in the supernatant of ELAVL 1-knockdown HepAD38 cells were lower than those in the control group (Fig. 2B). Similarly, western blottinghas demonstrated that ELAVL 1 knockdown in the stable Flag-HBXoverexpressing HepG2 cells obviously reduced Flag-HBx protein levels (Fig. 2C). Altogether, ELAVL1 plays a significant role in the regulation of not only HBV-derived RNA expression but also in its protein production.

\section{The role of ELAVL1 in cell proliferation ability}

The alteration of cell proliferation by ELAVL1 loss of function was then examined. Although the inhibition of HBV replication by the addition of tetracycline modestly weakened cell proliferation (Fig. 3A), lentiviral knockdown of ELAVL1 significantly repressed the cell growth in tetracycline-treated HepAD38 cells (Fig. 3B). These results indicate that ELAVL1 regulates the expression of not only viral proteins but also other tumor-related factors involved in cell proliferation.

\section{Expression of ELAVL1 in primary HCC tissues}

Next, the expression level of ELAVL1 in HCC tissues was then examined. Analyses of The Cancer Genome Atlas (TCGA) data have revealed that there are many cases in which the ELAVL1 mRNA levels were significantly higher in primary HCC tissues than in normal liver tissues (Fig. 4). To investigate the association between ELAVL1 expression and clinicopathological features, immunohistochemical analyses have been conducted on 77 paired samples consisting of tumor and adjacent nontumor tissues (Fig. 5A). ELAVL1 was expressed in the nuclei of hepatocytes at varying frequencies in nontumor tissues. Of the 77 nontumor samples, $37(48.1 \%)$ and 21 (27.3\%) were classified as partial expression ( $<50 \%$ of nuclei in hepatocytes) and diffuse expression ( $\geq 50 \%$ of nuclei in hepatocytes) patterns, respectively, but 19 (24.6\%) demonstrated no expression of ELAVL1 (Fig. 5B). ELAVL1 expression was observed in the nuclei of cholangiocytes but not of infiltrating lymphocytes in the portal area. In primary HCC tissues, 7 (9.1\%), $23(29.9 \%)$, and $47(61.0 \%)$ of the 77 samples were classified as negative, partial expression ( $<50 \%$ of nuclei in HCC cells), and diffuse expression ( $\geq 50 \%$ of nuclei in HCC cells) patterns, respectively. Among 47 HCCs with diffuse expression patterns, 13 cases (16.9\%) have demonstrated ELAVL1 expression not only in the nuclei but also in the cytoplasm of HCC cells. From the perspective of background liver disease, the ELAVL1 positivity rates in HBV-related HCC $(93.8 \%)$ are higher than those in HCV-related (89.3\%) and nonviral HCC (90.9\%). However, no significant difference was observed among these groups.

Patient characteristics, including between ELAVL1 $1^{\text {low }} \mathrm{HCC}$ and ELAVL1 $1^{\text {high }} \mathrm{HCC}$, were then compared (Table 1). Considering other clinical parameters, including age, gender, fibrosis, AFP levels, BCLC stages, and Edmondson-Steiner grades, no significant differences were also observed between ELAVL $1^{\text {low }}$ HCC and ELAVL1 ${ }^{\text {high }} \mathrm{HCC}$. In addition, no clinical factors that correlated with the ELAVL1-positivity were observed in HBV-related HCC (Table 2).

Subsequently, prognostic analyses using the Kaplan-Meier method were then conducted according to the ELAVL1 expression. No significant difference in recurrence-free survival (RFS) has been observed in 
all ELAVL $1^{\text {low }}$ and ELAVL1 ${ }^{\text {high }} \mathrm{HCC}$ patients $(p=0.080)$ (Fig. $\left.5 \mathrm{C}\right)$. Although no significant difference was found in the RFS between ELAVL $1^{\text {low }}$ and ELAVL $1^{\text {high }} \mathrm{HCC}$ patients, even in those with HBV-related HCC, a trend showing that the RFS in ELAVL1 $1^{\text {low }}$ HCC patients was better than that in ELAVL1 ${ }^{\text {high }}$ HCC patients was observed $(p=0.061)$. Notably, no recurrence was observed in patients with ELAVL low HCC (Fig. 5D). Together, it might be that the defective expression of ELAVL1 acts to prevent the recurrence of HBVrelated HCC.

\section{Discussion}

NAs, which are widely used for the treatment of chronic HBV infection worldwide, suppress HBV replication by inhibiting the reverse transcription of pregenomic RNA to HBV-DNA ${ }^{14}$. Oral treatment with NAs has a deterrent effect on HCC development through serum HBV-DNA level reduction and liver function improvement ${ }^{15,16}$. In fact, cases of HCC development even when the serum HBV-DNA level is below the detection sensitivity were reported. Although NA has an excellent inhibitory effect on HBV replication, its direct inhibitory effect on cccDNA is limited. The cccDNA in the liver tissue performs a central role as a viral persistent reservoir in HBV infection ${ }^{17}$. Therefore, viral mRNA transcription and viral protein translation using cccDNA as a template usually persist under NA treatment. In fact, the cancer incidence rate has been reported to be higher in HBV patients with high hepatitis B core-related antigen (HBcrAg) levels in the blood, which is said to reflect the amount of cccDNA in the liver tissue, than in patients with low levels ${ }^{18}$. Therefore, cccDNA elimination is the ultimate goal of hepatitis $B$ treatment. Various drug developments have also been attempted. However, clinical application has not been achieved at this time ${ }^{19,20}$.

Considering the current status, inhibition of HBV-derived RNA, template for protein synthesis, and viral DNA replication may be an alternative. Generally, RNA is extremely unstable in vivo, but RNA-binding proteins can stabilize RNA by binding to specific RNA sequences and can contribute to a smooth transcriptional regulation ${ }^{21,22}$. Previously, ELAVL1 was successfully demonstrated to bind to HBV-RNAs, including the $S, X$, and core regions ${ }^{12}$. In this study, the role of ELAVL1 in the transcriptional mechanism of HBV has been investigated.

Expectedly, but importantly, our in vitro assays demonstrated that the transcription of HBV pgRNA driven by tetracycline depletion in HepAD38 cells resulted in increased ELAVL1 mRNA levels. Concordant with these results, the expression level of ELAVL1 has been reported to increase in HepG2 and Hep3B cells with forced expression of $\mathrm{HBx}^{23}$. Subsequently, a marked suppression of HBV-RNA transcription was observed in our loss-of-function assay of ELAVL1. Furthermore, the amounts of HBs protein in HepAD38 cells and $\mathrm{HBx}$ protein in HepG2 cells were also significantly decreased after ELAVL 1 knockdown. These results indicated that ELAVL1 may be crucial for stable RNA transcription and protein synthesis of HBV.

Among the proteins encoded by the HBV viral genome, HBx specifically affects the transcription of various cancer-related genes and enhances cell proliferation and colony-forming ability 24,25 . Indeed, in 
the present study, the proliferative activity of tetracycline-treated HepAD38 cells with HBV-RNA transcription off was lower than that of tetracycline-depleted cells with HBV-RNA transcription on. ELAVL 1 knockdown in tetracycline-treated HepAD38 cells with HBV-RNA transcription off further reduced proliferative capacity. ELAVL1 has been reported to regulate the transcription of growth factors, including c-myc, c-fos, and c-jun, and cyclins, including cyclin A, B1, E, and D 126,27 . These findings indicated that ELAVL1 could regulate the cell proliferation ability of HCC cells in HBV replication-dependent and HBV replication-independent manners. Altogether, ELAVL1 is a potent therapeutic target for the inhibition of not only HBV replication but also of HCC cell growth.

It is well known that ELAVL1 protein translocates from the cell nuclei (predominantly localized) to the cytoplasm in response to proliferative and stress stimuli, increasing the half-life and/or modulating the translation rate of target mRNAs ${ }^{28,29}$. Both an increase in ELAVL1 expression levels and its localization to cytoplasm are closely associated with malignant transformation ${ }^{30,31}$. Concordant with these findings, our immunohistochemical analyses revealed that 70/77 (90.9\%) of HCC samples were positive for ELAVL1. The staining strength for ELAVL1 of the nuclei in HCC tissues was stronger than that in adjacent nontumor tissues in most cases. Although $13 \mathrm{HCC}$ tissues exhibited not only nuclear expression but also cytoplasmic expression of ELAVL1, only two cases demonstrated coexpression of ELAVL1 in the nontumor tissues.

Previous studies have demonstrated that higher ELAVL1 expression is closely associated with unfavorable prognosis in various cancer types ${ }^{32,33}$. Unexpectedly but importantly, no statistical differences in RFS were observed between ELAVL $1^{\text {high }}$ and ELAVL $1^{\text {low }}$ HCC patients. When limited to HBV patients, ELAVL $1^{\text {low }}$ HCC patients exhibited favorable RFS compared with ELAVL1 ${ }^{\text {high }}$ patients. These results indicate that ELAVL1 plays a significant role in the development and recurrence of HBV-related HCC. Conversely, cytoplasmic ELAVL1 expression has been reported to be associated with aggressive tumor phenotype ${ }^{34,35}$. Based on these results, this study also focused on the 13 cases with both nuclear and cytoplasmic ELAVL1 expressions, but no special clinical features were observed. Further analyses would be necessary in a larger number of HCC samples.

Pharmacological disruption of ELAVL1 would be very important for clinical application. Currently, the ELAVL1 function has multiple inhibitors, and these compounds are classified into two main categories based on their action ${ }^{36}$. One is drugs that inhibit the cytoplasmic transfer of ELAVL1, namely, MS-444, Nbenzylcantharidinamide, Latrunculin A, and Blebbistatin. The others are small molecule compounds, including CMLD-2 and KH3, which inhibit ELAVL1 binding to target mRNAs. These drugs have been reported to inhibit the growth, metastasis, and invasion of cancer cells mainly during preclinical studies. Preclinical and clinical trials have yet to be conducted to validate their anti-HBV effects.

In conclusion, ELAVL1 was successfully demonstrated to contribute to not only HBV replication but also to HCC cell growth. It may be a potent therapeutic target for the treatment of HBV-related HCC. 


\section{Materials And Methods}

Cell culture

HepAD38 cells, expressing pgRNA under the control of the inducible tetracycline promoter, were cultured in D-MEM/Ham's F-12 culture medium (Merck, Darmstadt, Germany) supplemented with $10 \%$ fetal bovine serum with or without $1 \mathrm{mg} / \mathrm{mL}$ of tetracycline to maintain the repression of HBV expression or HBV replication, respectively ${ }^{37,38}$. Flag-HBX-overexpressed HepG2 cells were cultured in Dulbecco's Modified Eagle Medium (Merck, Darmstadt, Germany) supplemented with $10 \%$ fetal bovine serum ${ }^{39}$.

\section{Quantitative real-time PCR}

The total RNA of cells was extracted using RNeasy Mini Kit (Qiagen, Valencia, CA, USA). Quantitative realtime PCR was performed with an MX3000P qPCR system (Stratagene, San Diego, CA, USA) using the Universal Probe Library System (Roche Diagnostics, Mannheim, Germany) or TB Green ${ }^{\text {TM }}$ Premix Ex Taq ${ }^{\text {TM }}$ II (Tli RNaseH Plus) Kit (Takara Bio Inc., Shiga, Japan) according to the manufacturer's protocols. The sequences of primers for human ELAVL 1 and GAPDH were as follows: ELAVL 1 (forward 5'CTGATGAATTCTCCCTTGTTCC-3', reverse 5'-GGCTTGGCAAATTACACTGAA-3') and GAPDH (forward 5'CTGACTTCAACAGCGACACC-3', reverse 5'-TAGCCAAATTCGTTGTCATACC-3'). The sequences of primers for total HBV mRNA, which amplified the region from nucleotides (nt) 1803 to 1894 in HBV sequences covering all HBV transcripts (3.5-, 2.4-, 2.1-, and 0.7-kb mRNAs), and HBV 3.5-kb mRNA, which amplified the region from nt 2268 to 2390 in HBV sequences, were as follows ${ }^{40}$ : total HBV mRNA (sense 5'TCACCAGCACCATGCAAC-3', antisense 5'-AAGCCACCCAAGGCACAG-3') and HBV 3.5-kb mRNA (sense 5'GAGTGTGGATTCGCACTCC-3', antisense 5'-GAGGCGAGGGAGTTCTTCT-3').

\section{Western blotting}

HCC cells were subjected to western blot analyses ${ }^{40}$. Briefly, the lysates were separated by $8 \%$ SDS-PAGE gels (Bio-Rad Laboratories, Inc.) and were then transferred to a polyvinylidene difluoride membrane (BioRad Laboratories, Inc.). The membranes were blotted with primary antibodies against ELAVL1 (Cell Signaling Technology), tubulin (Oncogene Science, Cambridge, MA, USA), and Flag (DYKDDDDK) tag (Wako Pure Chemical Industries, Osaka, Japan) and horseradish peroxidase (HRP)-conjugated secondary antibody (GE Healthcare Life Sciences). The membranes were developed using Immobilon western Chemiluminescent HRP Substrate (EMD Millipore), and the signals were detected using ChemiDoc XRS Systems (Bio-Rad Laboratories, Inc.).

\section{Measurement of HBs antigen levels}

The HBs antigen levels in culture media were measured using chemiluminescent enzyme immunoassay (CLEIA, Lumipulse System; Fujirebio, Tokyo, Japan).

\section{Lentiviral production and transduction}


Lentiviral vectors (CS-H1-shRNA-EF-1a-EGFP) expressing short hairpin RNA (shRNA) targeting human ELAVL 1 (target sequence: sh-ELAVL 1-1, 5'-GGTTTGGGCGGATCATCAACT-3'; sh-ELAVL 1-2, 5'-

GGTTTGGCTTTGTGACCATGA-3') and Iuciferase ( $L U C$ ) were constructed. Recombinant lentiviruses were produced as described previously ${ }^{41}$. The cells were transduced using a lentiviral vector in the presence of protamine sulfate $(10 \mu \mathrm{g} / \mathrm{mL}$; Sigma-Aldrich, St. Louis, MO, USA).

\section{Cell proliferation assay}

Cells $\left(1 \times 10^{4}\right.$ cells/well) were seeded on 96-well plates and cultured for $96 \mathrm{~h}$. MTS assays were performed using the CellTiter Aqueous One Solution Kit (Promega Corporation, Madison, WI, USA) according to the manufacturer's protocols. Cell viability was assessed at 24, 48, 72, and $96 \mathrm{~h}$ and was defined as a percentage of the absorbance measured in the control cells. The dye of the formazan product resulting from MTS reduction was quantified using an automatic multiwell spectrophotometer, namely, InfiniteF50 (Tecan, Männedorf, Switzerland).

\section{Patients and surgical specimens}

A total of 77 pairs of tumor and adjacent nontumor tissues were subjected to clinicopathological analyses. Written informed consent was obtained from all patients. Paraffin-embedded tumor sections and the surrounding nontumor tissues were examined via H\&E staining and immunohistochemistry with anti-ELAVL1 antibody (Cell Signaling Technology). Based on the ELAVL1 expression in the nuclei of cells, HCC and nontumor tissues were classified as follows: negative, partial expression ( $<50 \%$ of nuclei), and diffuse expression ( $\geq 50 \%$ of nuclei). HCCs with negative or partial ELAVL1 expression were classified as the ELAVL1 ${ }^{\text {low }}$ group, whereas HCCs with diffuse ELAVL1 expression were classified as the ELAVL 1 high group. All patients received postoperative radiological follow-up every 2-6 months. Radiological assessments were evaluated based on the response evaluation criteria in solid tumors ${ }^{42}$. This study was approved by the research ethics committees of the Graduate School of Medicine, Chiba University (approval number: 3300) and performed according to the Declaration of Helsinki.

\section{Data collection and analysis from The Cancer Genome Atlas (TCGA)-Liver Hepatocellular Carcinoma (LIHC)}

RNA sequencing (RNA-seq) datasets (ID: TCGA.LIHC.sampleMap/HiSeqV2) were downloaded using the UCSC Xena Browser (https://xenabrowser.net/). The RNA sequencing dataset shows the gene-level transcription estimates as in normalized log counts per million (logCPM). In total, secondary analyses have been conducted on RNA-seq data from both the normal liver $(n=50)$ and primary HCC $(n=371)$.

\section{Statistical analysis}

Data are expressed as mean with standard deviation (SD) or median with minimum to maximum and interquartile range (IQR). Statistical differences in the quantitative variables between groups were determined using either Student's t-test or the Mann-Whitney Utest. Chi-squared test was used for 
categorical variables. The log-rank test was used to analyze survival data. The level of significance was set to $p<0.05$. All statistical analyses have been conducted using the SPSS statistical software version 27 (IBM, Chicago, IL, USA).

\section{Declarations}

\section{Acknowledgements:}

The authors thank Risa Kakiuchi and Anna Sakaue for technical assistance.

\section{Author Contribution:}

H.K. and T.C. designed the study. H.K., T.C., T.I., K.O., M.N., K.F., H.U., T.I., T.S., N.F., K.K., K.K., N.K., S.K., M.N., T.K., T.S., R.N., S.O., R.M., S.N., T.K. and H.M. collected samples. H.K., T.C., T.K., J.A., N.Q., Y.M., M.K., Y.K., Y.Z. and K.S. analyzed and interpreted the data. H.K. and T.C. performed statistical analyses and prepared the manuscript. J.K., A.K., M.O., M.O. and N.K. edited and reviewed the manuscript.

\section{Financial support:}

This study was partially supported by the Program for Basic and Clinical Research on Hepatitis from Japan Agency for Medical Research and Development (AMED, \#JP20fk0210054).

\section{Conflict of Interest:}

The authors declare no competing financial interests.

\section{References}

1. Tang, L. S. Y., Covert, E., Wilson, E. \& Kottilil, S. Chronic hepatitis B infection: A Review. JAMA, 319, 1802-1813 (2018).

2. WHO. Global Hepatitis Report 2017.

3. Degenhardt, L. et al. Estimating the burden of disease attributable to injecting drug use as a risk factor for HIV, hepatitis C, and hepatitis B: findings from the Global Burden of Disease Study 2013. Lancet Infect Dis, 16, 1385-1398 (2016).

4. Levrero, M. et al. Control of cccDNA function in hepatitis B virus infection. J Hepatol, 51, 581-592 (2019).

5. Bock, C. T. et al. Structural organization of the hepatitis B virus minichromosome. J Mol Biol, 307, 183-196 (2001).

6. Seeger, C. \& Mason, W. S. Hepatitis B virus biology. Microbiol Mol Biol Rev, 64, 51-68 (2000).

7. Takata, A. et al. Mutual antagonism between hepatitis B viral mRNA and host microRNA let-7. Sci Rep, 6, 23237 (2016). 
8. Liang, H. W. et al. Hepatitis B virus-human chimeric transcript HBx-LINE1 promotes hepatic injury via sequestering cellular microRNA-122. J Hepatol, 64, 278-291 (2016).

9. Duriez, M. et al. Alternative splicing of hepatitis B virus: A novel virus/host interaction altering liver immunity. J Hepatol, 67, 687-699 (2017).

10. Marcellin, P. et al. Tenofovir disoproxil fumarate versus adefovir dipivoxil for chronic hepatitis B. $N$ Engl J Med, 359, 2442-2455 (2008).

11. Hadziyannis, S. J. et al. Adefovir dipivoxil for the treatment of hepatitis B e antigen-negative chronic hepatitis B. N Engl J Med, 348, 800-807 (2003).

12. Sekiba, K. et al. DHX9 regulates production of hepatitis B virus-derived circular RNA and viral protein levels. Oncotarget, 9, 20953-20964 (2018).

13. Meisner, N. C. et al. mRNA openers and closers: modulating AU-rich element-controlled mRNA stability by a molecular switch in mRNA secondary structure., 5, 1432-1447 (2004).

14. Tang, L. S. Y. et al. A Review. JAMA, 319, 1802-1813 (2018).

15. Liaw, Y. F. et al. Lamivudine for patients with chronic hepatitis B and advanced liver disease. N Eng J Med, 351, 1521-1531 (2004).

16. Hosaka, T. et al. Long-term entecavir treatment reduces hepatocellular carcinoma incidence in patients with hepatitis B virus infection., 58, 98-107 (2013).

17. Xia, Y. \& Guo, H. Hepatitis B virus cccDNA: Formation, regulation and therapeutic potential. Antiviral Res, 180, 104824 (2020).

18. Tada, T. et al. HBcrAg predicts hepatocellular carcinoma development: An analysis using timedependent receiver operating characteristics. J Hepatol, 65, 48-56 (2016).

19. Martinez, M. G., Villeret, F., Testoni, B. \& Zoulim, F. Can we cure hepatitis B virus with novel directacting antivirals? Liver Int, 40 (Suppl 1), 27-34 (2020).

20. Martinez, M. G. et al. Covalently closed circular DNA: The ultimate therapeutic target for curing HBV infections. J Hepatol, 75, 706-717 (2021).

21. Ross, J. mRNA stability in mammalian cells. Microbiol Rev, 59, 423-450 (1995).

22. Zubiaga, A. M. \& Greenberg, M. E. The nonamer UUAUUUAUU is the key AU-rich sequence motif that mediates mRNA degradation. Mol Cell Biol, 15, 2219-2230 (1995).

23. Hung, C. M. et al. Hepatitis B virus $X$ upregulates HuR protein level to stabilize HER2 expression in hepatocellular carcinoma cells. Biomed Res Int. 2014, 827415 (2014).

24. Zhang, X. D., Wang, Y. \& Ye, L. H. Hepatitis B virus X protein accelerates the development of hepatoma. Cancer Biol Med, 11, 182-190 (2014).

25. Slagle, B. L. \& Bouchard, M. J. Role of HBx in hepatitis B virus persistence and its therapeutic implications. Curr Opin Virol, 30, 32-38 (2018).

26. Wang, J. et al. Multiple functions of the RNA-binding protein HuR in cancer progression, treatment responses and prognosis. Int J Mol Sci, 14, 10015-10041 (2013). 
27. Pascale, A. \& Govoni, S. The complex world of post-transcriptional mechanisms: is their deregulation a common link for diseases? Focus on ELAV-like RNA-binding proteins. Cell Mol Life Sci, 69, 501517 (2012).

28. Wang, W., Caldwell, M. C., Lin, S., Furneaux, H. \& Gorospe, M. HuR regulates cyclin A and cyclin B1 mRNA stability during cell proliferation. EMBO J, 19, 2340-2350 (2000).

29. Wang, W., Yang, X., Cristofalo, V. J., Holbrook, N. J. \& Gorospe, M. Loss of HuR is linked to reduced expression of proliferative genes during replicative senescence. Mol Cell Biol, 21, 5889-5898 (2001).

30. López de Silanes, I., Lal, A. \& Gorospe, M. HuR: post-transcriptional paths to malignancy. RNA Biol, 2 , 11-13 (2005).

31. Kotta-Loizou, I., Giaginis, C. \& Theocharis, S. Clinical significance of HuR expression in human malignancy. Med Oncol, 31, 161 (2014).

32. Yi, X., Zhou, Y., Zheng, W. \& Chambers, S. K. HuR expression in the nucleus correlates with high histological grade and poor disease-free survival in ovarian cancer. Aust NZ J Obstet Gynaecol, 49, 93-98 (2009).

33. Yoo, P. S. et al. Tissue microarray analysis of 560 patients with colorectal adenocarcinoma. high expression of HuR predicts poor survival. Ann Surg Oncol, 16, 200-207 (2009).

34. Heinonen, M. et al. Cytoplasmic HuR Expression Is a Prognostic Factor in Invasive Ductal Breast Carcinoma. Cancer Res, 65, 2157-2161 (2005).

35. Miyata, Y. et al. High expression of HuR in cytoplasm, but not nuclei, is associated with malignant aggressiveness and prognosis in bladder cancer. PLoS One, 8, e59095 (2013).

36. Papatheofani, V. et al. HuR Protein in Hepatocellular Carcinoma: Implications in Development, Prognosis and Treatment. Biomedicines, 9, 119 (2021).

37. Kakizaki, M. et al. The immunological function of extracellular vesicles in hepatitis B virus-infected hepatocytes. PLoS One, 13, e205886 (2018).

38. Ladner, S. K. et al. Inducible expression of human hepatitis B virus (HBV) in stably transfected hepatoblastoma cells: a novel system for screening potential inhibitors of HBV replication. Antimicrob Agents Chemother, 41, 1715-1720 (1997).

39. Sekiba, K. et al. Inhibition of HBV Transcription From cccDNA With Nitazoxanide by Targeting the HBx-DDB1 Interaction. Cell Mol Gastroenterol Hepatol, 7, 297-312 (2019).

40. Imai, Y. et al. Interferon-y induced PD-L1 expression and soluble PD-L1 production in gastric cancer. Oncol Lett, 20, 2161-2168 (2020).

41. Chiba, T. et al. The polycomb gene product BMI1 contributes to the maintenance of tumor-initiating side population cells in hepatocellular carcinoma. Cancer Res, 68, 7742-7749 (2008).

42. Eisenhauer, E. A. et al. New response evaluation criteria in solid tumours: revised RECIST guideline (version 1.1). Eur J Cancer, 45, 228-247 (2009).

\section{Tables}


Table 1. Clinical features of ELAVL1 $1^{\text {low }}$ and ELAVL $1^{\text {high }}$ HCC patients

\begin{tabular}{lccr}
\hline Characteristics & $\begin{array}{c}\text { ELAVL1 }^{\text {low }} \\
(\mathrm{n}=30)\end{array}$ & $\begin{array}{c}\text { ELAVL1 } \\
(\mathrm{n}=47)\end{array}$ & $p$-value \\
\hline Age (years) (median (IQR)) & $67(11)$ & $70(13)$ & 0.257 \\
Gender: male/female & $23 / 7$ & $37 / 10$ & 0.832 \\
Etiology: HBV/HCV/others & $6 / 12 / 12$ & $10 / 16 / 21$ & 0.866 \\
Fibrosis stage: CH/LC & $25 / 5$ & $34 / 13$ & 0.266 \\
AFP (ng/ml) (median (IQR)) & $12.9(206.7)$ & $9.2(116.9)$ & 0.703 \\
BCLC stage: A/B & $27 / 3$ & $38 / 9$ & 0.280 \\
Edmondson-Steiner grade: I/II/II/IV & $2 / 8 / 16 / 4$ & $1 / 11 / 26 / 9$ & 0.699 \\
\hline
\end{tabular}

Abbreviations: $\mathrm{HCC}$, hepatocellular carcinoma; $\mathrm{HBV}$, hepatitis $\mathrm{B}$ virus; $\mathrm{HCV}$, hepatitis $\mathrm{C}$ virus; $\mathrm{CH}$, chronic hepatitis; LC, liver cirrhosis; AFP, alpha-fetoprotein; BCLC, Barcelona clinic liver cancer

Table 2. Clinical features of ELAVL1 $1^{\text {low }}$ and ELAVL $1^{\text {high }}$ HBV-related HCC patients

Characteristics

Age (years) (median (IQR))

Gender: male/female

Fibrosis stage: $\mathrm{CH} / \mathrm{LC}$

$\operatorname{AFP}(\mathrm{ng} / \mathrm{mL})$ (median (IQR))

BCLC stage: A/B

Edmondson-Steiner grade: I/II/III/IV
ELAVL1 $1^{\text {low }}$

$(\mathrm{n}=6)$

66 (12)

$4 / 2$

$5 / 1$

$662.7(1651.7)$

$5 / 1$

$0 / 0 / 3 / 3$
ELAVL1 ${ }^{\text {high }}$

$p$-value

$(\mathrm{n}=10)$

$62(15)$

0.713

$10 / 0$

0.051

$8 / 2$

0.869

$3.9(268.7)$

0.263

$8 / 2$

0.869

0.309

Abbreviations: HBV, hepatitis B virus; $\mathrm{HCC}$, hepatocellular carcinoma; $\mathrm{CH}$, chronic hepatitis; $\mathrm{LC}$, liver cirrhosis; AFP, alpha-fetoprotein; BCLC, Barcelona clinic liver cancer

\section{Figures}



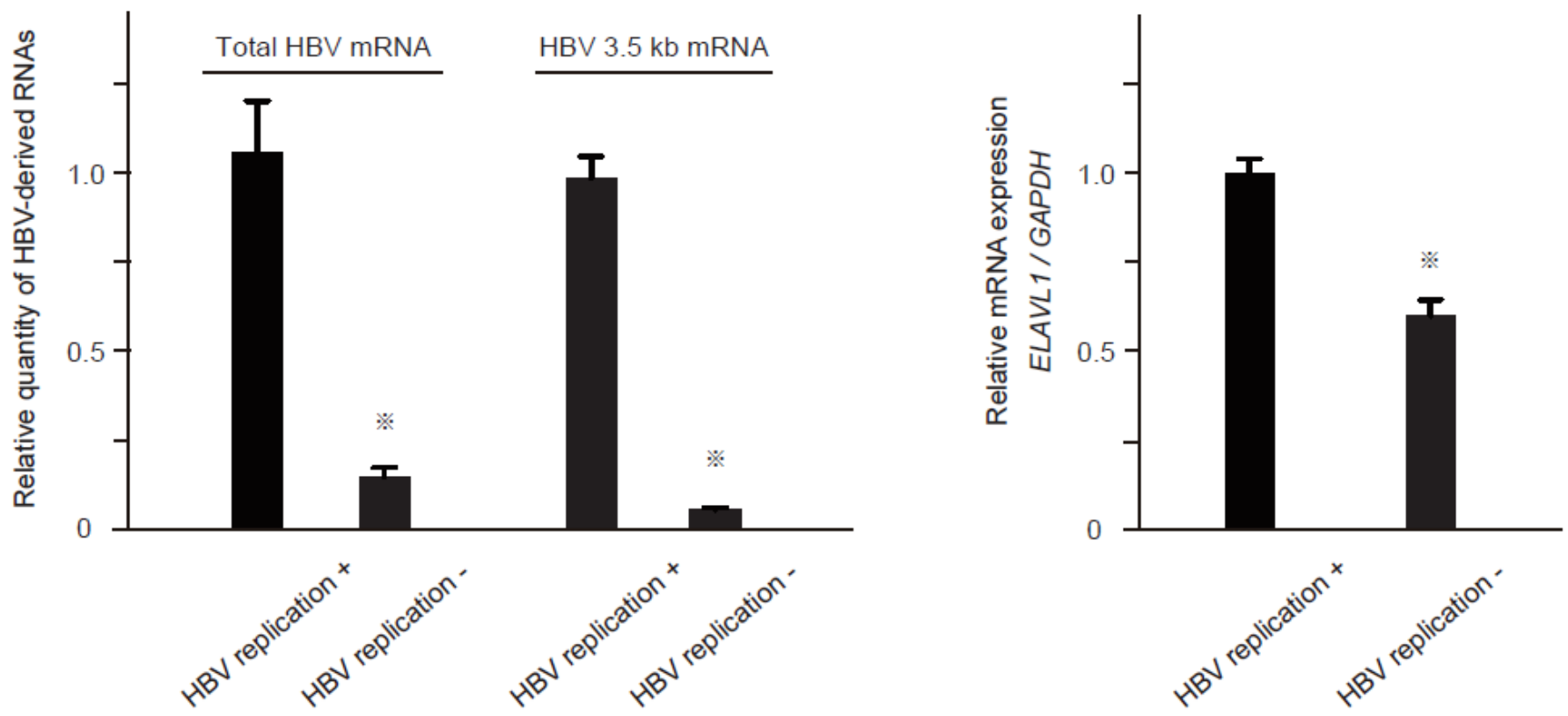

C

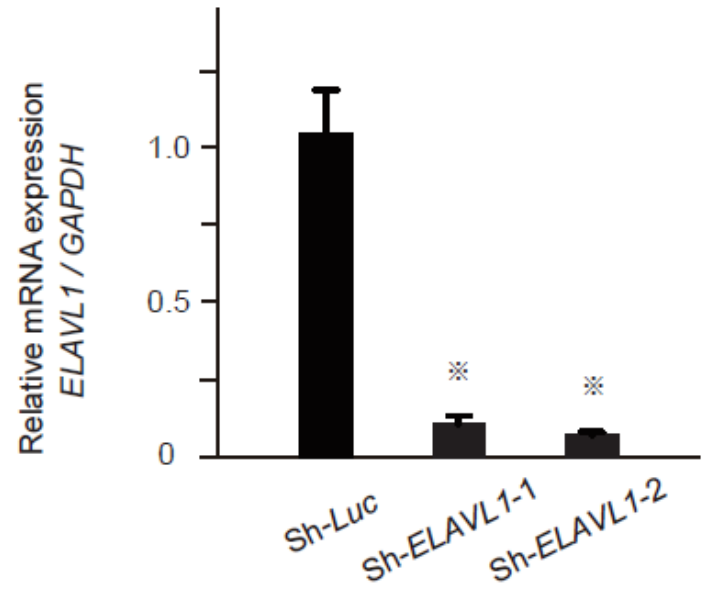

D

HepAD38

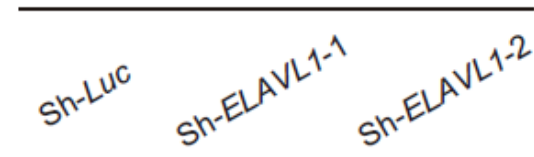

ELAVL1

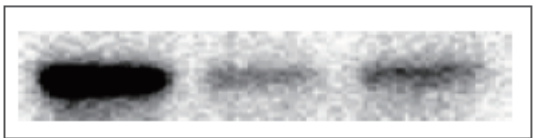

$-30 \mathrm{kDa}$

Tubulin

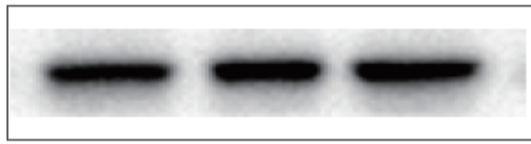

\section{Figure 1}

ELAVL1-knockdown assays in HCC cells. (A) Total HBV mRNA and 3.5-kb mRNA levels of HepAD38 cells in the presence or absence of tetracycline were determined via quantitative real-time PCR analyses. (B) The HepAD38 cells in the presence or absence of tetracycline were subjected to quantitative real-time PCR analyses for ELAVL1 mRNA expression. (C) ELAVL1-knockdown HepAD38 cells in the presence of tetracycline were subjected to quantitative real-time PCR analyses for ELAVL1 mRNA expression. (D) HepAD38 cells with stable knockdown of ELAVL1 were subjected to western blot analyses using antiELAVL1 and antitubulin antibody (loading control). 
A

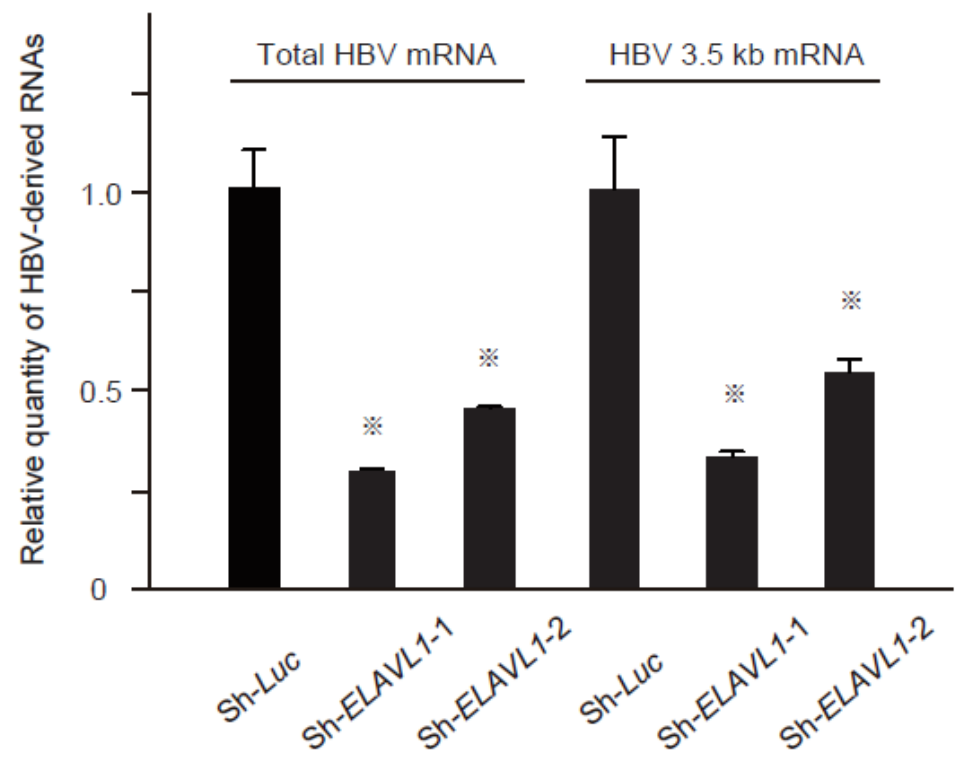

C

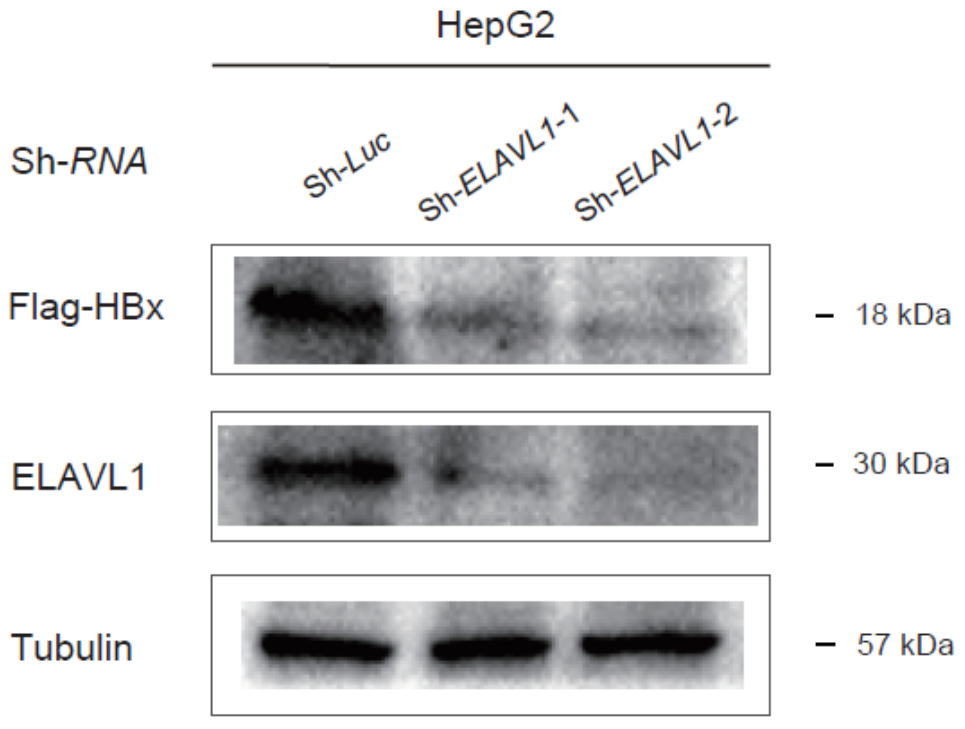

B

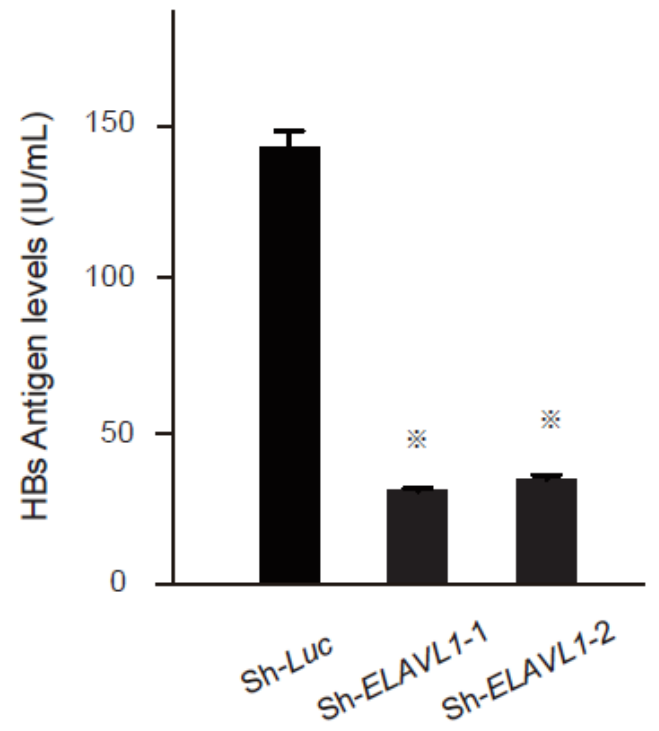

\section{Figure 2}

In vitro assays of HepAD38 cells. (A) Total HBV mRNA and 3.5-kb mRNA levels of ELAVL1-knockdown HepAD38 cells in the absence of tetracycline were determined via quantitative real-time PCR analyses. (B) Supernatants derived from ELAVL1-knockdown HepAD38 cells in the absence of tetracycline were subjected to CLEIA for HBs antigen measurement. (C) ELAVL1-knockdown HepG2 cells that constitutively express Flag-HBx protein were subjected to western blot analyses using anti-Flag, anti-ELAVL1, and antitubulin antibodies (loading control). 
A

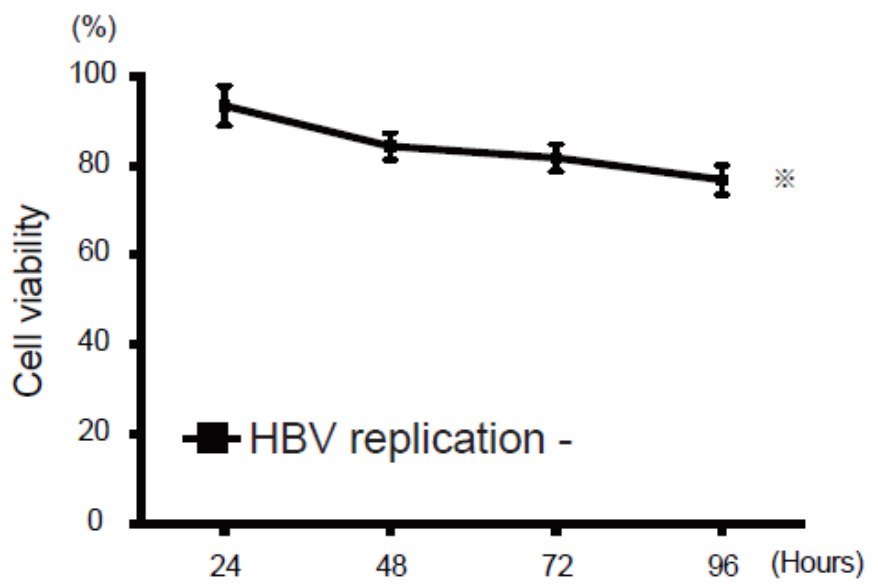

B

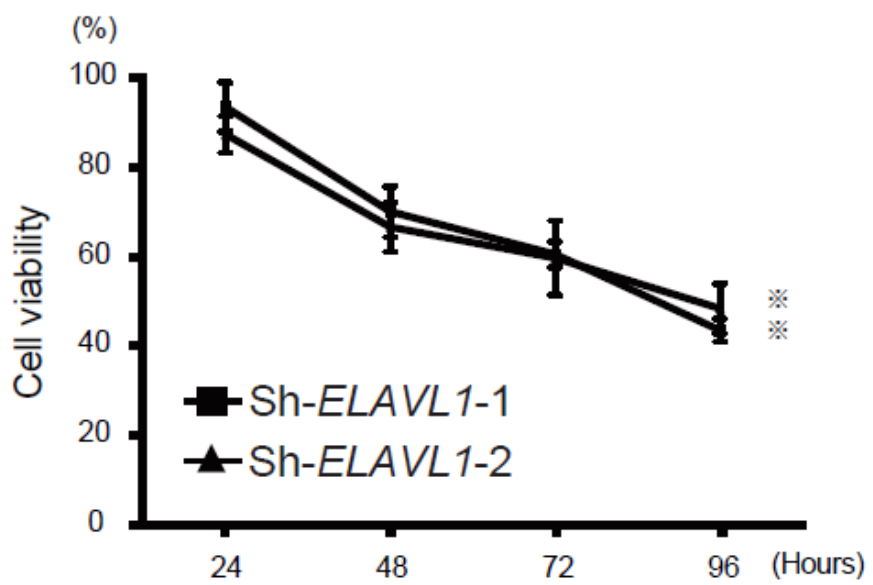

\section{Figure 3}

Cell growth inhibition in ELAVL1-knockdown HepAD38 cells. (A) Decreased cell growth abilities in HepAD38 cells in the presence of tetracycline compared with those in the absence of tetracycline. (B) Cell growth inhibition in ELAVL1-knockdown HepAD38 cells in the presence of tetracycline. 


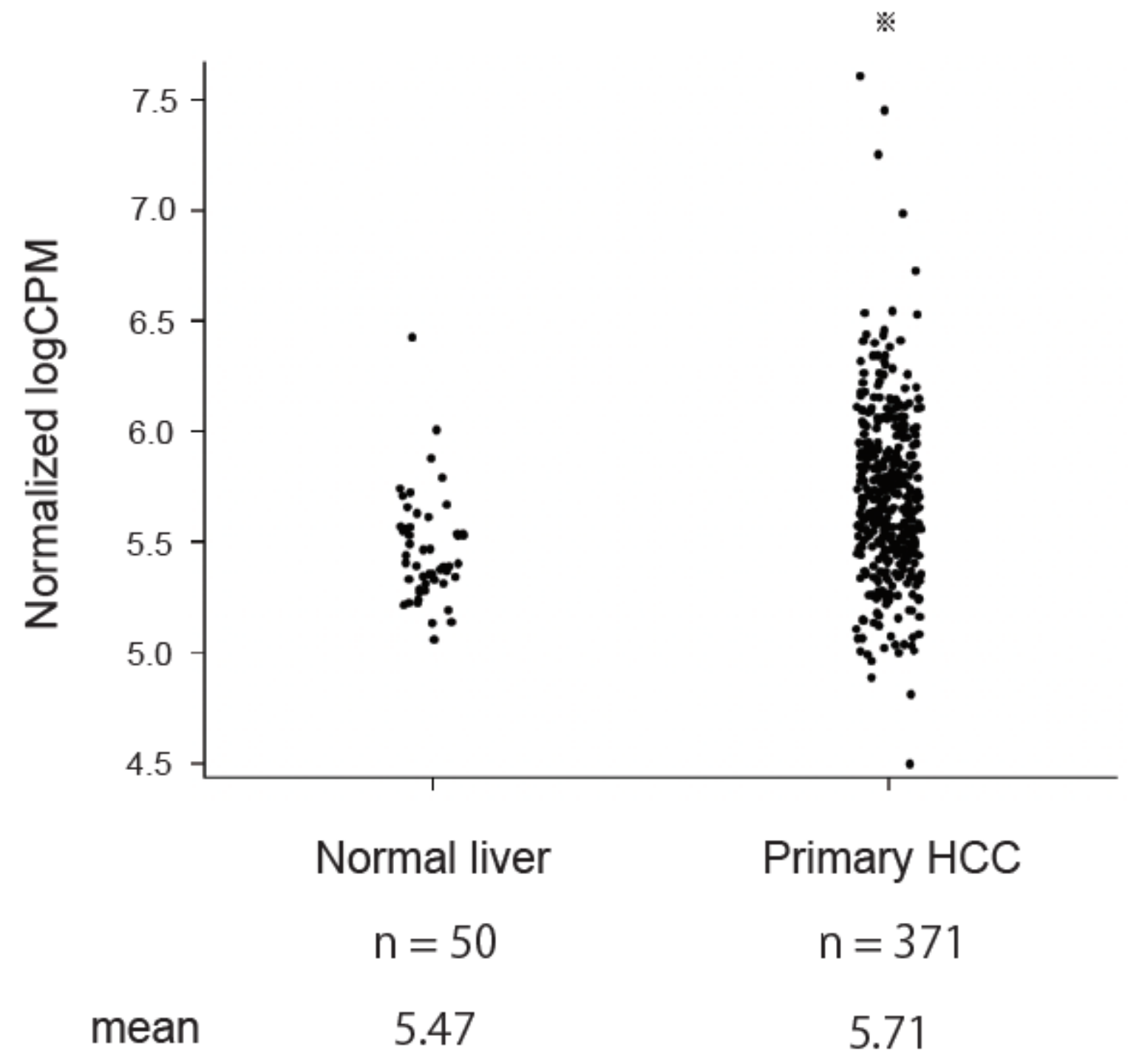

Figure 4

ELAVL1 expression in normal liver and HCC in the TCGA database. ELAVL1 mRNA expression based on the dataset obtained from TCGA-LIHC. 
A
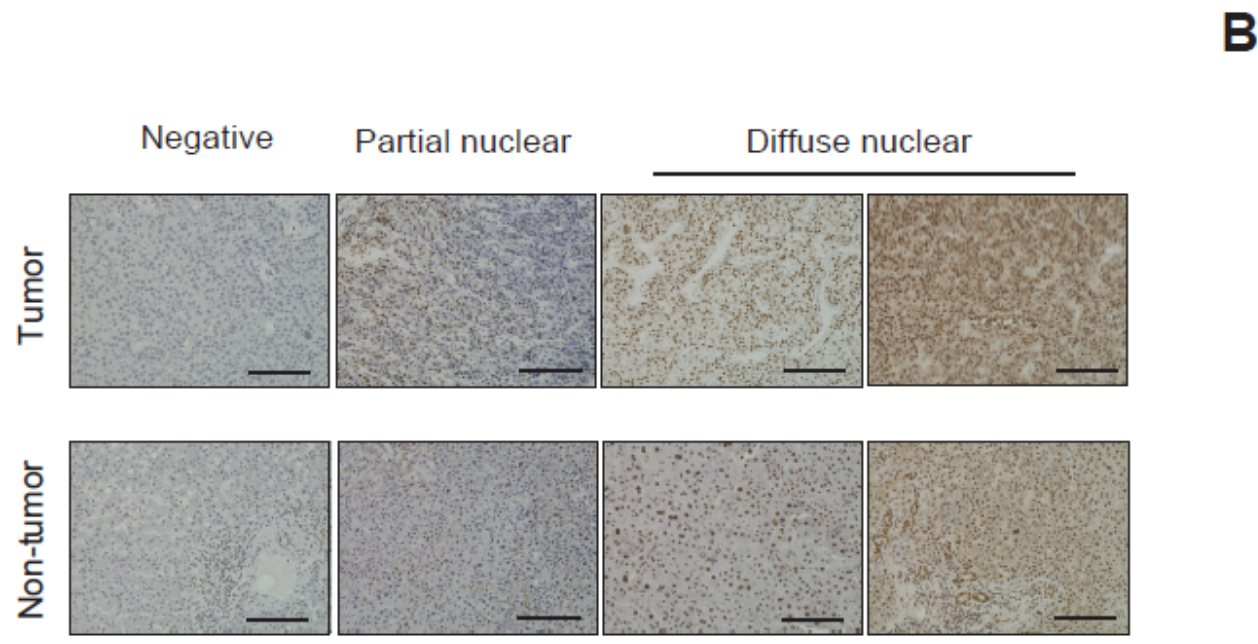

B

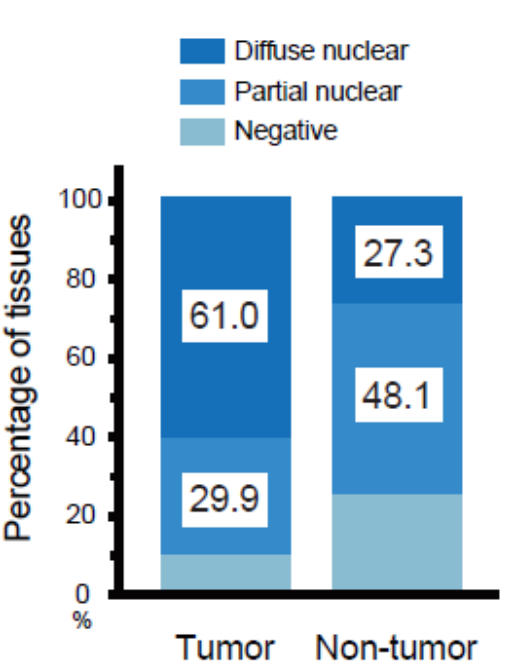

C
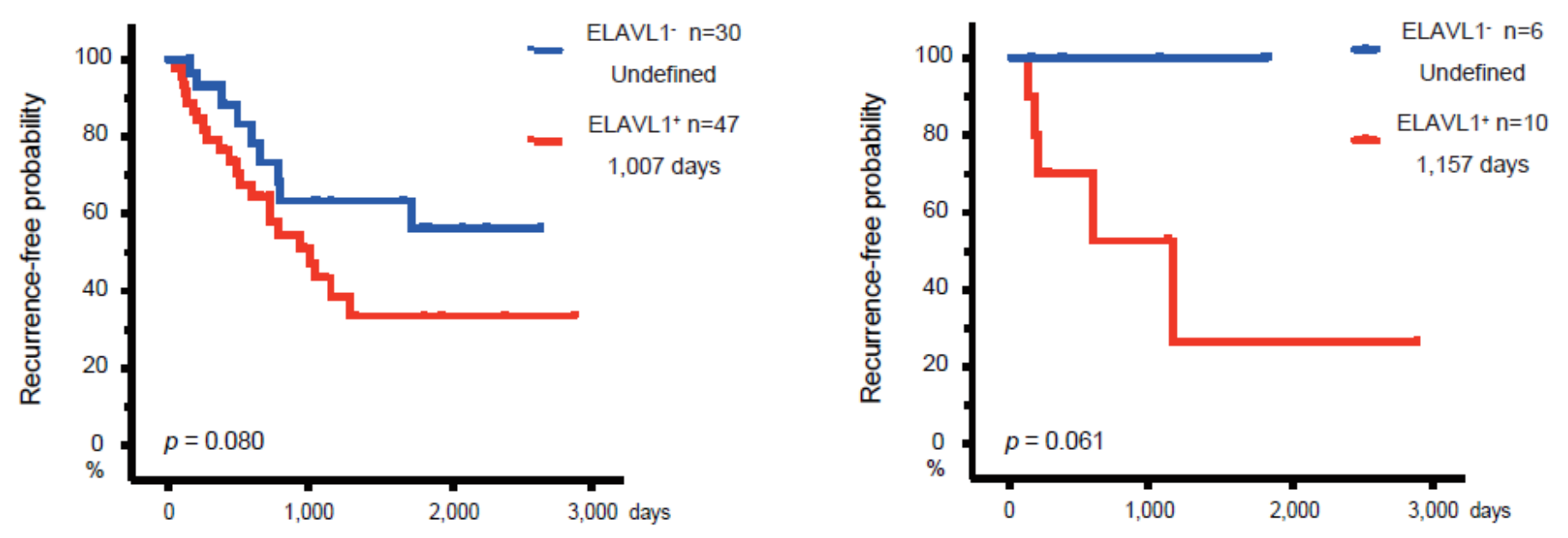

\section{Figure 5}

Clinicopathological analyses of ELAVL1 in HCC surgical samples. (A) Representative ELAVL1 immunohistochemistry analyses for tumor and adjacent surrounding nontumor tissues. Scale bar $=100$ $\mu \mathrm{m}$. (B) Calculation of ELAVL1 expression patterns in tumor and adjacent surrounding nontumor tissues. (C) Cumulative progression-free survival based on the ELAVL1 expression in all HCC patients $(n=77)$. (D) Cumulative RFS rate based on the ELAVL1 expression in HBV-related HCC patients $(n=16)$.

\section{Supplementary Files}

This is a list of supplementary files associated with this preprint. Click to download.

- Supplementaryinformation.docx 